\title{
Meningitis bacteriana de presentación atípica en paciente adulta mayor: reporte de caso
}

\section{Atypical presentation for bacterial meningitis in an elderly woman: report of a case}

Correspondencia

Fanny Elizabeth Ramírez Calderón Jr. Domingo Cueto $N^{\circ} 120$

Jesús María. Lima, Perú

Teléfono: 5112654967 anexo 15313

racaelifa@hotmail.com

Recibido: 10/02/2019

Arbitrado por pares

Aprobado: 06/03/2019

Citar como: Ramírez-Calderón F, Sotelo-Jiménez P, RodriguezMalaver C. Meningitis bacteriana de presentación atípica en paciente adulta mayor: reporte de caso. Acta Med Peru. 2019;36(1):62-7

\section{Fanny Ramírez-Calderón ${ }^{1 a}$, Pedro Sotelo-Jiménez ${ }^{1 b}$, Carlos Rodriguez-Malaver ${ }^{1 c}$}

1 Departamento de Emergencia, Hospital Edgardo Rebagliati Martins. Lima, Perú.

a Médico de emergencias, b Médico internista, c Médico cirujano

\section{RESUMEN}

La meningitis es un proceso inflamatorio de las leptomeninges cuyas manifestaciones clínicas son inespecíficas en los extremos de la vida. Presentamos el caso de una paciente mujer de 70 años de edad, que ingresó a emergencia con un tiempo de enfermedad de 14 horas y un cuadro de cefalea, vómitos y trastorno del sensorio, hemiparesia derecha en mal estado general; se diagnosticó inicialmente un accidente cerebro vascular. La tomografía cerebral no mostró evidencia de lesiones isquémicas o hemorrágicas. La paciente empeoró y cursó con fiebre, por lo que se realizó una punción lumbar y se inició tratamiento antibiótico empírico. El cuadro empeoró y la paciente falleció a las 36 horas del ingreso. Tanto el cultivo del líquido cefalorraquideo como el hemocultivo fueron positivos para Streptococcus pneumoniae. Discutimos el diagnóstico, presentación atípica, tratamiento y la necesidad del reconocimiento oportuno de esta patología.

Palabras clave: Meningitis bacteriana; Streptococcus pneumoniae; Adulto mayor (fuente: DeCS BIREME).

\begin{abstract}
Meningitis is an inflammatory process affecting leptomeninges, whose clinical manifestations are nonspecific both in the very young and the elderly. We present the case of a 70-year-old female patient who was admitted to emergency with 14-hour time of illness who presented with headache, vomiting, conscience impairment, and right hemiparesis. The patient was in a poor general condition, and the initial diagnosis was stroke. The brain CT scan showed no evidence of ischemic or hemorrhagic lesions. The patient worsened and developed fever, so a lumbar puncture was performed and empirical antibiotic treatment was started. Her condition got worse, and she died 36 hours after admission. Both the cerebrospinal fluid culture and the blood culture were positive for Streptococcus pneumoniae. We discuss the diagnosis, the atypical presentation, treatment and the need for the timely recognition of this condition.
\end{abstract}

Keywords: Meningitis, bacterial; Streptococcus pneumonia; Elderly (source: MeSH NLM). 


\section{INTRODUCCIÓN}

La meningitis aguda (MA) es una emergencia neurológica que requiere una evaluación y tratamiento inmediato ${ }^{[1]}$. Su incidencia es cinco casos por cada 100000 adultos por año y se estima que a nivel mundial hay anualmente 1,2 millones de casos ${ }^{[2]}$.

El proceso inflamatorio de las leptomeninges, característico de esta patología, se evidencia en el líquido cefaloraquideo (LCR) a través de una pleocitosis a predominio de poliforfonucleares, hiperproteinorráquia e hipoglucorráquia, hallazgos que son claves para establecer el diagnóstico.

En el curso de la enfermedad se pueden cumplir criterios de sepsis, sepsis grave y shock séptico que sumado a la clínica reflejan la gravedad. Asimismo, las complicaciones y la mortalidad de los servicios de emergencia, en donde se atienden estos pacientes, continúan siendo altos en relación al tiempo de estancia hospitalaria.

La tríada clásica fiebre, rigidez de nuca y alteración del sensorio suele presentarse aunque no en todos los casos ${ }^{[2]}$. Por otro lado, las manifestaciones clínicas son inespecíficas en las edades extremas de la vida ${ }^{[3,4]}$.

El sospechar de MA de origen bacteriano (MAB) continúa siendo un reto por la dificultad para aislar al agente patógeno mediante el cultivo. Son dos bacterias las que se encuentran en un $80 \%$ de los casos (Streptococcus pneumoniae [SP] seguido de Neisseria meningitidis [NM] $)^{[1,2]}$. El SP es un diplococo grampositivo, afecta todas las edades -principalmente niños y adultos mayores en quienes causa infección severa-y es una de las principales causas de infección meníngea en todo el mundo ${ }^{[2,5]}$. La MAB causa una mortalidad cercana al $30 \%$ a $37 \%$ y provoca secuelas neurológicas en el $52 \%$ de los sobrevivientes ${ }^{[5,6]}$.

Presentamos un caso de meningitis bacteriana de presentación atípica en una paciente adulta mayor con evolución no favorable; este reporte de caso fue elaborado siguiendo la guía CARE ${ }^{[7]}$.

\section{REPORTE DE CASO}

Paciente mujer de 70 años de edad, natural y procedente de Lima, Perú, de ocupación ama de casa. Negó la existencia de alergias, con antecedente de apendicectomía. Ingresó al servicio de emergencia del Hospital Nacional Edgardo Rebagliati Martins con un cuadro de cefalea, vómitos, trastorno del sensorio, un tiempo de enfermedad de 14 horas, afebril.

\section{Hallazgos clínicos}

A la inspección, la paciente se encontraba en mal estado general, deshidratada, delgada, somnolienta, con palidez de piel y mucosas. En el examen físico se midió una presión arterial (PA) de 138/75 mmHg, una frecuencia cardiaca (FC) de 120 latidos por minuto (lat/min), una frecuencia respiratoria (FR) de 18 respiraciones por minuto (resp/min), saturación de oxígeno al $95 \%$ y temperatura axilar de $36,5^{\circ} \mathrm{C}$. En el examen preferencial se auscultaron ruidos cardiacos rítmicos sin presencia de soplos, roncus escasos en ambos campos pulmonares; a la palpación, abdomen blando, depresible, aunque impresionó una palpación dolorosa. En el examen neurológico, la paciente se mostraba confusa, con la mirada desviada a la derecha, pupilas reactivas, hemiparesia derecha, sin rigidez de nuca.

\section{Evaluación diagnóstica}

Con lo hallado se estableció el diagnóstico presuntivo de accidente cerebro vascular más neumonía aspirativa. Los estudios de laboratorio (Tabla 1) reportaron, en el hemograma: leucocitos 8,16 k/ul, linfocitos 9,2 k/ul, neutrófilos $88,3 \%, \mathrm{Hb}$ $13,1 \mathrm{~g} / \mathrm{dl}$, plaquetas $114 \mathrm{k} / \mathrm{ul}$; creatinina 0,8 mg/dl, glucosa 106 $\mathrm{mg} / \mathrm{dl}$, urea 29,9 mg/dl. La radiografía de tórax (Figura 1A) reveló tractos fibrosos en el ápice de ambos campos pulmonares, lo que indicaría el antecedente de tuberculosis. La tomografía cerebral (TC) no mostró lesiones agudas ni sangrado (Figura 1C).

A la reevaluación, nueve horas luego del ingreso, las pupilas eran isocóricas y reactivas; sin embargo, la paciente no obedecía a órdenes simples, emitía sonidos incomprensibles e incoherentes, además reaccionaba a la movilización de extremidades que era escasa; Babinsky negativo, rigidez de nuca, reflejos cutáneo plantar y flexor bilateral conservados. Por tanto, se planteó el diagnóstico presuntivo de accidente cerebro vascular versus meningitis. Se solicitó interconsulta a Neurología quienes indicaron la presencia de paratonía cervical y ausencia rigidez de nuca, por lo cual se solicitó una nueva TC.

Luego de 20 horas del ingreso, la paciente mostró signos de descompensación, presentó una PA de 112/53 mmHg, FC de $158 \mathrm{lat} / \mathrm{min}, \mathrm{FR}$ de $40 \mathrm{resp} / \mathrm{min}$, temperatura de $38,3^{\circ} \mathrm{C}$, escala de Glasgow 8 puntos (ocular: 2 puntos, verbal: 2 puntos, motor: 4 puntos), pupilas de $3 \mathrm{~mm}$ poco reactivas y rigidez de nuca. La nueva tomografía mostró borramiento de surcos, sin signos de lesiones isquémicas o hemorrágicas (Figura 1D). Se procedió a protección de vía aérea más ventilación mecánica y punción lumbar (PL). Se actualizó el diagnóstico a insuficiencia respiratoria aguda, shock séptico de foco neurológico versus respiratorio, meningitis aguda y trastorno hidroelectrolítico (Tabla 1).

Tanto el estado hemodinámico con del sensorio de la paciente fueron empeorando hasta concluir en el fallecimiento. EI diagnóstico final fue de meningitis aguda; además, se obtuvo el resultado positivo para Streptococcus pneumoniae en el cultivo de LCR y en el hemocultivo (sensible a penicilina, vancomicina y cloranfenicol).

\section{Intervención terapéutica}

Al ingreso, la paciente recibió reposición hídrica con cristaloides, $50 \mathrm{mg}$ de dimenhidrinato por vía endovenosa (EV) y $40 \mathrm{mg}$ de omeprazol EV. A las 20 horas de su ingreso, debido al empeoramiento del cuadro, se inició el uso de imipenen (500 

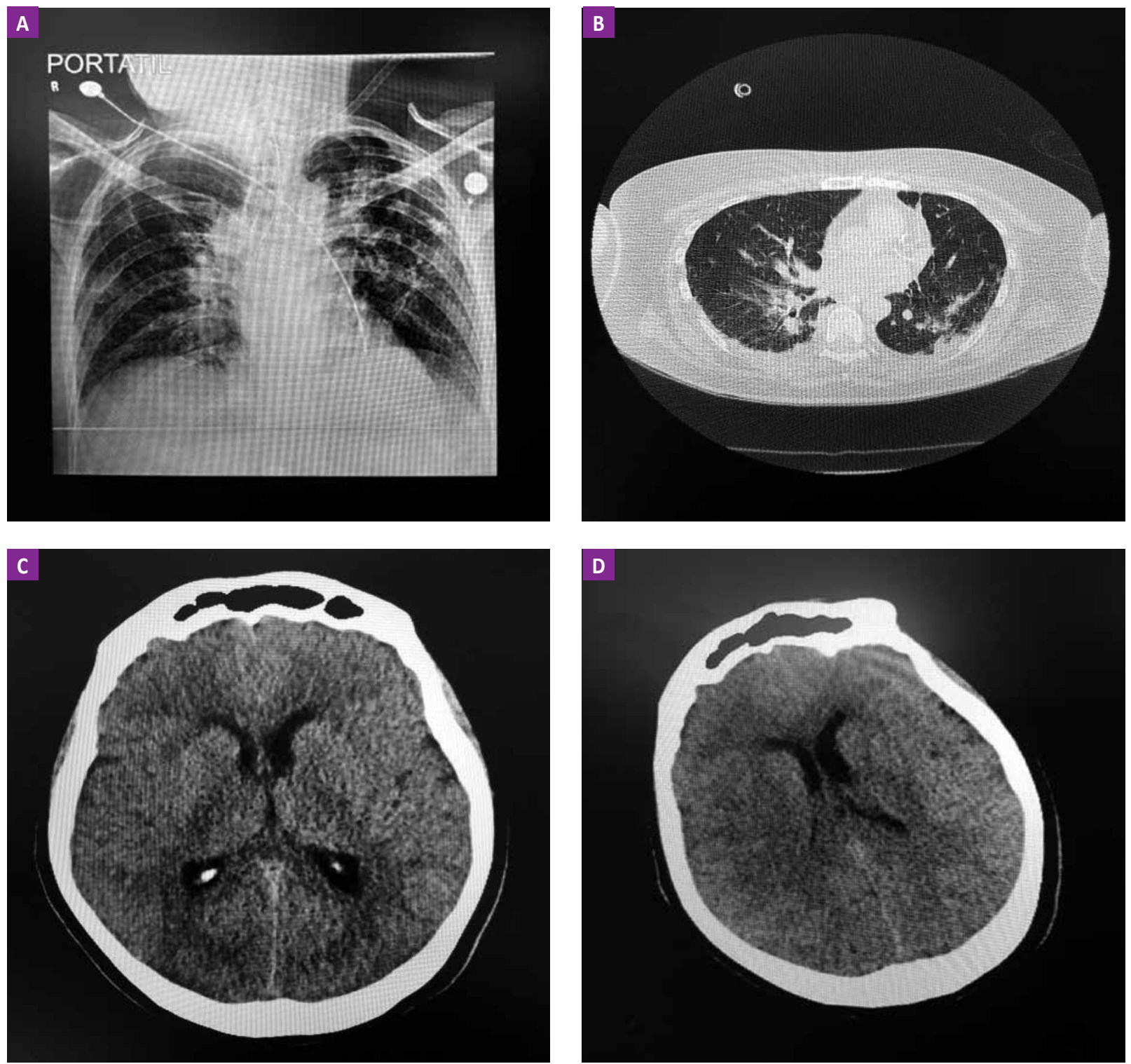

FIGURA 1. A. Radiografía torácica que muestra tractos fibrosos secuelares en ambos ápices de los pulmones. No consolidación. B. Tomografía pulmonar que muestra pulmones en hipoventilación, tractos fibrosos secuelares pleuroparenquimales en ambos ápices, incluyen bronquiectasias y nódulos cálcicos. También atelectasias en ambas bases y anteriores en lóbulo superior izquierdo. Cambios hipoventilatorios en declive y consolidación en espacio aéreo con broncograma en base derecha atelectasia versus inflamatoria asociada, no nódulos sospechosos de malignidad. Derrame pleural laminar izquierdo. C. Tomografía cerebral al ingreso que el que el parénquima supra e infratentorial muestra leves cambios involutivos. No lesiones isquémicas ni hemorrágicas agudas definidas. No focos de edema cerebral. Linea media central. No hidrocefalea ni colecciones hemorrágicas yuxtadurales. D. Segunda tomografía cerebral. Se descarta evento isquémico o hemorrágico reciente. No colecciones intra ni extraxiales. Llama la atención la disminución de surcos y cisuras, pudiendo corresponder a edema difuso.

$\mathrm{mg}$ EV c/6 h), vancomicina (1 g EV c/12 h), dexametasona (4 $\mathrm{mg}$ ) y administración de oxígeno. La paciente persistió con mala evolución y compromiso hemodinámico, a las 22 horas del ingreso se colocó tubo endotraqueal, se indicó uso de ventilación mecánica asistida controlada y se procedió a colocar catéter venoso central (CVC). Se administró infusión de dopamina a $10 \mathrm{~cm}^{3} / \mathrm{h}$ y noradrenalina $15 \mathrm{~cm}^{3} / \mathrm{h}$ lento y diluido; se cambió de antibiótico a ceftriaxona ( $2 \mathrm{gEV} \mathrm{c} / 12 \mathrm{~h}$ ), vancomicina ( $1 \mathrm{gEV} \mathrm{c} / 12 \mathrm{~h}$ ), aciclovir (750 mg EV c/8 h) y metilprednisolona (1 g EV en pulsos).

\section{Seguimiento y resultados}

Al ingreso, la paciente presentó trastorno del sensorio, cefalea, vómitos y focalización; sin rigidez de nuca ni otros signos meníngeos, con una TC sin evidencia de isquemia o hemorragia (Figura 1C) y exámenes de laboratorio no contributorios (Tabla 1).

A las 20 horas de su ingreso presentó fiebre y rigidez de nuca, se amplió la anamnesis con familiares quienes referían que había presentado alza térmica y sido medicado en un policlínico 
Tabla 1. Resultados de laboratorio.

\begin{tabular}{|c|c|c|}
\hline Prueba & Resultado & Referencia \\
\hline \multicolumn{3}{|l|}{ Hemograma } \\
\hline Leucocitos & $8,16 \mathrm{k} / \mathrm{ul}$ & $4,00-11,00$ \\
\hline Neutrófilos & $88,3 \%$ & $40-70$ \\
\hline Linfocitos & $9,2 \%$ & $19-48$ \\
\hline Monocitos & $1,1 \%$ & $3,4-9$ \\
\hline Neutrófilos absolutos & $7,29 \mathrm{k} / \mathrm{ul}$ & $1,8-8$ \\
\hline Linfocitos absolutos & $0,75 \mathrm{k} / \mathrm{ul}$ & $0,9-5,2$ \\
\hline Monocitos absolutos & $0,09 \mathrm{k} / \mathrm{ul}$ & $0,16-0,95$ \\
\hline Hemoglobina & $13,1 \mathrm{~g} / \mathrm{dl}$ & $12-16$ \\
\hline Hematocrito & $36,7 \%$ & $37-47$ \\
\hline Plaquetas & $114 \mathrm{k} / \mathrm{ul}$ & $130-400$ \\
\hline Gr porcentual & $1 \%$ & \\
\hline Grupo sanguíneo & A negativo & \\
\hline PCR & ------ & \\
\hline \multicolumn{3}{|l|}{ Bioquímica y electrolitos } \\
\hline Creatinina & $0,65 \mathrm{mg} / \mathrm{dl}$ & $0,5-0,8$ \\
\hline Glucosa & $163 \mathrm{mg} / \mathrm{dl}$ & $74-106$ \\
\hline Urea & $29,9 \mathrm{mg} / \mathrm{dl}$ & $22-55$ \\
\hline Potasio & $3,28 \mathrm{mg} / \mathrm{dl}$ & $3,5-5,5$ \\
\hline Sodio & $138 \mathrm{mg} / \mathrm{dl}$ & $132-146$ \\
\hline Cloro & $102 \mathrm{mg} / \mathrm{dl}$ & $99-109$ \\
\hline \multicolumn{3}{|l|}{ Perfil Hepático } \\
\hline Proteínas totales & $7,9 \mathrm{~g} / \mathrm{dl}$ & $5,7-8,2$ \\
\hline Albúmina & $4,3 \mathrm{~g} / \mathrm{dl}$ & $3,2-4,8$ \\
\hline Globulina & $3,6 \mathrm{~g} / \mathrm{dl}$ & $2-3,5$ \\
\hline TGO & $30 \mathrm{U} / \mathrm{L}$ & $0-34$ \\
\hline TGP & $20 \mathrm{U} / \mathrm{L}$ & $10-49$ \\
\hline Bilirrubina total & $1,1 \mathrm{mg} / \mathrm{dl}$ & $0,3-1,2$ \\
\hline Bilirrubina directa & $0,3 \mathrm{mg} / \mathrm{dl}$ & -- \\
\hline Bilirrubina indirecta & $0,8 \mathrm{mg} / \mathrm{dl}$ & -- \\
\hline Fosfatasa alcalina & $69 \mathrm{U} / \mathrm{L}$ & $45-129$ \\
\hline \multicolumn{3}{|l|}{ Perfil de Coagulación } \\
\hline Tiempo de Protrombina & $12,6 \mathrm{~s}$ & $11-13,5$ \\
\hline TTP & $27 \mathrm{~s}$ & $25-37$ \\
\hline $\mathrm{TT}$ & $16 \mathrm{~s}$ & $17-22$ \\
\hline Fibrinógeno & $439 \mathrm{mg} / \mathrm{dl}$ & $200-400$ \\
\hline \multicolumn{3}{|l|}{ Líquido Cefalorraquídeo } \\
\hline Color & $\begin{array}{l}\text { Ligeramente } \\
\text { xantocrómico }\end{array}$ & \\
\hline Aspecto & Transparente & \\
\hline Glucosa & $0 \mathrm{mg} / \mathrm{dl}$ & $45-80$ \\
\hline Proteínas totales & $936,2 \mathrm{mg} / \mathrm{dl}$ & $15-40$ \\
\hline \multicolumn{3}{|l|}{ Gases Arteriales } \\
\hline $\mathrm{pH}$ & 7,33 & \\
\hline $\mathrm{PCO}_{2}$ & 28,5 & \\
\hline $\mathrm{HCO}_{3}$ & $16,6 \mathrm{mmHg}$ & \\
\hline Sat $\mathrm{O}_{2}$ & -- & \\
\hline $\mathrm{PO}_{2}$ & 48,5 & \\
\hline $\mathrm{Lac}$ & $7,1 \mathrm{mmol} / \mathrm{l}$ & \\
\hline $\mathrm{FiO}_{2}$ & 21 & \\
\hline
\end{tabular}

PCR: proteína C reactiva; TGO: aspartato aminotransferasa; TGP: alanina aminotransferasa; TTP: tiempo de tromboplastina parcial; TT: tiempo de trombina privado antes de su ingreso a emergencia; la nueva TC mostró borramiento de surcos -a descartar edema- (Figura 1D) y se inició terapia antibiótica (imipenen y vancomicina) y dexametasona previa PL, en dicho examen se obtuvo un líquido amarillo citrino, con presión aumentada, glucosa cero e hiperproteinorraquia $(936,2 \mathrm{mg} / \mathrm{dl})$ (Tabla 1). Fue diagnosticada como meningitis aguda y evaluada por Neurología e Infectología.

A las 22 horas, se indicó intubación orotraqueal, ventilación mecánica y colocación de CVC, la paciente persistió con mala evolución por lo que se inició uso de vasopresores y se amplió la cobertura antibiótica. Los resultados de cultivo de LCR y hemocultivo fueron positivos para Streptococcus pneumoniae. La paciente falleció a las 36 horas de su ingreso con diagnóstico de meningitis aguda.

\section{DISCUSIÓN}

Alrededor del 12 a $24 \%$ de las atenciones en un servicio de emergencias corresponde a pacientes adultos mayores, de ellos el $49 \%$ son mayores de 65 años, como el caso reportado; esta población se caracteriza por tener presentaciones atípicas de la enfermedad y suelen estar acompañadas de patologías crónicas complicadas ${ }^{[8]}$.

La evaluación geriátrica integral (EGI) es un proceso interdisciplinario necesario para identificar problemas físicos, sociales, funcionales y psíquicos que ayudarían a establecer un diagnóstico precoz. Dentro de esta evaluación, existen pruebas que por sus propias características (p.e. la complejidad de su realización) no pueden ser utilizados en emergencias, como ocurrió en nuestro caso. Esto nos lleva a comprender la necesidad de que dicha evaluación sea realizada pero previa a la atención en emergencias, es decir en el primer nivel de atención, justo antes de enfermar. Durante el manejo de la emergencia, estas pruebas serían poco útiles por el nivel de inestabilidad clínica del paciente, su grado de escolaridad o el desgaste e incomodidad que se produce al aplicarlas ${ }^{[8,9]}$.

Nuestra paciente presentó un cuadro clínico no característico MA y de rápida evolución, por lo cual el diagnóstico y tratamiento fueron tardíos, lo que limitó el manejo terapéutico e impidió la reversión del curso natural de la enfermedad.

La MAB puede presentar edema cerebral y presión intracraneal elevada resultando en herniación cerebral que condiciona a alta morbilidad y mortalidad ${ }^{[10]}$. La MAB por S. pneumoniae es una de las principales causas de infección meníngea en todo el mundo ${ }^{[11,12]}$.

De los pacientes con meningitis, de 11 a $19 \%$ está en coma al momento de la admisión, 29 a $42 \%$ con déficit neurológico y 7 a $21 \%$ presentan crisis convulsivas, lo que demuestra la gravedad de la enfermedad. El deterioro del paciente puede ser rápido y muy difícil de predecir; así el daño sistémico, como presión baja y taquicardia ocurren frecuentemente y son factores de mal pronóstico ${ }^{[6]}$, tal como se presentó en nuestra paciente. 
Los síntomas y signos clásicos como cefalea, fiebre, rigidez de nuca y trastorno del sensorio aislados -como fue en nuestro casoen la historia clínica tienen un valor diagnóstico bajo; en cambio la tríada de cefalea, fiebre y trastorno del sensorio tienen una sensibilidad diagnóstica de entre el 99 y $100 \%{ }^{[13]}$.

La ausencia de rigidez de nuca no es una regla que descarte la MAB por lo que debe prestarse mucha atención en aquellos pacientes con sospecha de neumonía y en ancianos ${ }^{[14]}$; nuestra paciente era una persona anciana con patología respiratoria, lo que generó distracción y una inoportuna definición del problema. El diagnóstico diferencial entre infección del sistema nervioso central (SNC) y otros diagnósticos será definido por el examen del LCR ${ }^{[15]}$. Así, un estudio realizado en Suecia encontró que la eliminación de las contraindicaciones para realizar una PL: empeoramiento del trastorno del sensorio o la ausencia de una TC previa, estuvo asociada con un diagnóstico temprano y resultados favorables ${ }^{[10]}$.

Ante la sospecha de MAB también debe hacerse un diagnóstico diferencial con desordenes sistémicos del SNC que podrían disminuir la intensidad de los signos y síntomas ${ }^{[16]}$ como sucedió en nuestra paciente. En un estudio realizado por Talan et al. demostraron que el $90 \%$ del tiempo en que se demoraba dar el tratamiento antibiótico ocurría luego de la evaluación médica, con variación en función de la gravedad de la enfermedad. Otros estudios mencionan que el manejo de pacientes asintomáticos con MA condiciona un retraso en el diagnóstico y es frecuente en pacientes ancianos ${ }^{[14]}$.

Así pues, en un estudio de 48 casos con diagnóstico de MAB se presentó demora mayor a dos días en el diagnóstico, con una mortalidad mayor de $50 \%$ y una etiología exclusivamente no meningocócica ${ }^{[3,14]}$; lo cual es análogo a nuestro caso. Otro estudio presentó una mortalidad menor a pesar de la demora en el inicio de tratamiento; aunque su población fue heterogénea y la etiología variada (bacteriana, viral, tuberculosa y criptococócica) ${ }^{[14]}$.

La utilización de corticoides como la dexametasona junto con los antibióticos en la MAB atenúa la respuesta inflamatoria y disminuye la morbimortalidad sobretodo en gérmenes como el neumococo ${ }^{[17]}$. El tratamiento empírico con cefalosporinas de tercera generación (ceftriaxona o cefotaxima) y vancomicina es el tratamiento de elección en la MAB ${ }^{[9,10]}$.

Una revisión sistemática encontró que la mortalidad es mayor en aquellas personas con MAB causada por Streptococcus pneumoniae (35\%), seguida por Haemophilus influenzae (25\%) y Neisseria meningitidis (4\%) ${ }^{[18,19]}$; nuestra paciente estuvo infectada por el primer patógeno y su desenlace fue fatal. La mortalidad también está asociada al inicio de antibiótico; un estudio demostró que la mortalidad aumenta $13 \%$ por cada hora se demoraba el uso de antibióticos ${ }^{[10]}$.

En nuestro caso, la MAB fue diagnosticada tardíamente debido a que nuestra paciente era de edad avanzada, en quienes la sintomatología simula otras enfermedades, se presenta atípica o asintomática, o con un inicio insidioso.

En conclusión, el diagnóstico tardío es una circunstancia común en $\mathrm{MAB}$ y puede asociarse a un cuadro clínico no característico en el momento de ingreso a emergencia; esto puede llevar a un tratamiento no adecuado y conducir a una mortalidad alta. Evaluar diferentes estrategias para minimizar la demora en el inicio de tratamiento deben ser evaluadas. La meningitis por SP en adultos y más aún en ancianos es una patología con alta morbilidad y mortalidad. Por lo tanto, es importante su rápido diagnóstico y tratamiento y aun así su desenlace puede ser rápido y mortal.

Agradecimientos: Al programa de mentoría implementado por el Instituto de Evaluación de Tecnologías en Salud e Investigación (IETSI) de EsSalud por el soporte para el desarrollo de este manuscrito.. A la doctora Lorena Oyanguren Serra por proporcionar los datos del paciente.

Contribuciones de autoría: FRC, PSJ y CRM han participado en la concepción y diseño del artículo y aprobación de la versión final del manuscrito. FRC elaboró el primer borrador del artículo. FRC, PSJ y CRM han realizado recolección de datos y la revisión crítica del contenido.

Fuentes de financiamiento: Ninguno.

Conflictos de interés: al momento de la concepción de la publicación del artículo los autores laboraban en EsSalud.

\section{REFERENCIAS BIBLIOGRÁFICAS}

1. Heckenberg SGB, Brouwer MC, van de Beek D. Bacterial meningitis. Handb Clin Neurol. 2014;121:1361-75.

2. Mora Mora LA, De Arco Espinosa ME, Plumet J, Micheli F. Meningitis bacteriana adquirida en la comunidad en mayores de 60 años. MEDICINA (Buenos Aires). 2015;75(6):367-72.

3. Morales-Casado MI, Julián-Jiménez A, Lobato-Casado P, CámaraMarín B, Pérez-Matos JA, Martínez-Maroto T. Factores predictores de meningitis bacteriana en los pacientes atendidos en urgencias. Enferm Infecc Microbiol Clin. 2017;35(4):220-8.

4. Nau R, Djukic M, Spreer A, Eiffert H. Bacterial meningitis: new therapeutic approaches. Expert Rev Anti Infect Ther. 2013;11(10):1079-95.

5. Martínez-Hernández L, Cornejo-Juárez P. Meningitis bacteriana aguda por S. pneumoniae. Med Interna Méx 2017;33(1):132-8.

6. McGill F, Heyderman RS, Panagiotou S, Tunkel AR, Solomon T. Acute bacterial meningitis in adults. Lancet. 2016;388(10063):3036-47.

7. Gagnier JJ, Kienle G, Altman DG, Moher D, Sox H, Riley D, et al. The CARE guidelines: consensus-based clinical case report guideline development. J Clin Epidemiol. 2014;67(1):46-51.

8. Porras JQ. Evaluación del paciente geriátrico en el servicio de urgencias. Rev Med Cos Cen. 2015;72(617):719-21.

9. Domínguez-Ardila A, García-Manrique JG. Valoración geriátrica integral. Aten Fam. 2014;21(1):20-3. 
10. Glimaker M, Johansson B, Grindborg O, Bottai M, Lindquist L, Sjolin J. Adult Bacterial Meningitis: Earlier Treatment and Improved Outcome Following Guideline Revision Promoting Prompt Lumbar Puncture. Clinical Infectious Diseases. 2015;60(8):1162-9.

11. Hasbun R, Tunkel AR. Central Nervous System Infections in the Elderly. En: Berhouma M, Krolak-Salmon P, editores. Brain and Spine Surgery in the Elderly . Cham: Springer; 2018. p. 81-94.

12. Rodríguez Peñil EG, Ojeda Carmona LE, Contreras Arenas AM, Díaz Alvarado AL. Meningitis bacteriana con presentación atípica: a propósito de un caso. Revista Médica Electrónica Portales Médicos [Internet]. 16 de noviembre de 2018 [citado 14 de nov 2018]. Disponible en: https://www.revista-portalesmedicos.com/revistamedica/envio-articulos-formato-pdf/

13. Aneja S. Acute Bacterial Meningitis - Early Diagnosis and Complications. Indian J Pediatr. 2015;82(4):303-5.

14. Bodilsen J, Brandt CT, Sharew A, Dalager-Pedersen M, Benfield T, Schønheyder HC, et al. Early versus late diagnosis in community- acquired bacterial meningitis: a retrospective cohort study. Clin Microbiol Infect. 2018;24(2):166-70.

15. Khatib U, van de Beek D, Lees JA, Brouwer MC. Adults with suspected central nervous system infection: A prospective study of diagnostic accuracy. J Infect. 2017;74(1):1-9.

16. Cunha BA. The clinical and laboratory diagnosis of acute meningitis and acute encephalitis. Expert Opin Med Diagn. 2013;7(4):343-64.

17. Borchorst S, Møller K. The role of dexamethasone in the treatment of bacterial meningitis - a systematic review: Dexamethasone for bacterial meningitis. Acta Anaesthesiol Scand. 2012;56(10):1210-21.

18. Prasad K, Singhal T, Jain N, Gupta PK. Third generation cephalosporins versus conventional antibiotics for treating acute bacterial meningitis. Cochrane Database Syst Rev. 2004;(2):CD001832.

19. Mayo Clinic. Encefalitis - Síntomas y causas [Internet]. Phoenix, AZ: Mayo Clinic; 2017 [citado 14 de nov 2018]. Disponible en: https:// www.mayoclinic.org/es-es/diseases-conditions/encephalitis/ symptoms-causes/syc-20356136

\title{
Ahora puede enviar sus artículos para Acta Médica Peruana en nuestro Open Journal System:
}

\author{
www.amp.cmp.org.pe
}

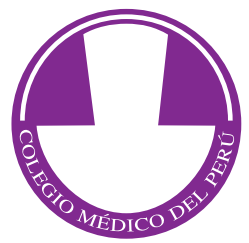

